\title{
Correction to: Pre-emptive use of Sorafenib combined with DLI post HSCT in AML FLT3+: a single center experience
}

Antonella Bruzzese - Giovanni Manfredi Assanto - Daniela Diverio - Luisa Quattrocchi - Daniela Carmini • Ursula La Rocca - Maria Stefania De Propris - Silvia Maria Trisolini - Mattia Brescini • Giulia Galassi • Walter Barberi • Anna Paola lori

Published online: 26 February 2021

(c) The Author(s), under exclusive licence to Springer Nature Limited 2021

Correction to: Bone Marrow Transplantation https://doi.org/10.1038/s41409-020-01174-w

The original version of this article unfortunately contained a mistake. The following correction has therefore been made in the original: The spelling of Ursula La Rocca's name was incorrect. The corrected author list is given above. The original article has been corrected. 